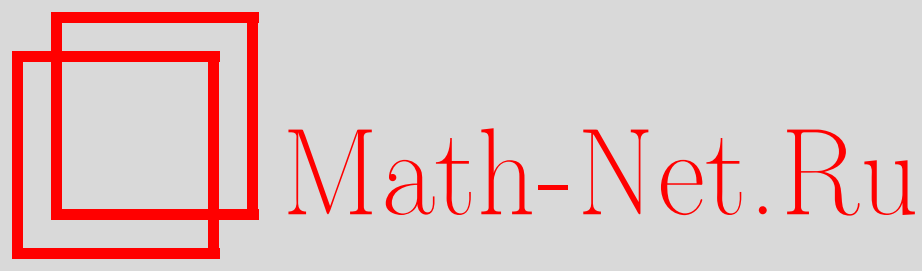

А. А. Панин, Локальная разрешимость и разрушение решения для уравнения Розенау-Бюргерса с различными граничными условиями, ТМФ, 2013, том 177, номер 1, 93-110

DOI: https://doi.org/10.4213/tmf8507

Использование Общероссийского математического портала Math-Net.Ru подразумевает, что вы прочитали и согласны с пользовательским соглашением http: //www.mathnet.ru/rus/agreement

Параметры загрузки:

IP : 54.224 .60 .19

26 апреля 2023 г., 13:18:37

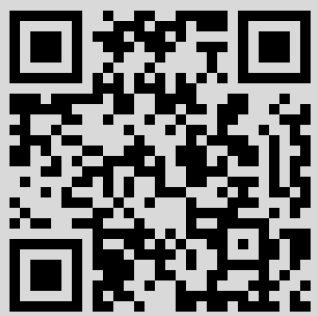




\section{ЛОКАЛЬНАЯ РАЗРЕШИМОСТЬ И РАЗРУШЕНИЕ РЕШЕНИЯ ДЛЯ УРАВНЕНИЯ РОЗЕНАУ-БЮРГЕРСА С РАЗЛИЧНЫМИ ГРАНИЧНЫМИ УСЛОВИЯМИ}

Рассмотрено несколько модельных начально-краевых задач для уравнения Розенау-Бюргерса с различными граничными условиями. Для каждой из задач доказана однозначная локальная разрешимость в классическом смысле, получены достаточное условие возникновения режима blow-up и оценка времени разрушения. При доказательстве используется известный метод пробных функций.

Ключевые слова: режим blow-up, локальная разрешимость, непродолжаемое решение, уравнение Розенау-Бюргерса.

DOI: $10.4231 / \operatorname{tmf} 8507$

\section{1. ВВЕДЕНИЕ}

Рассмотрим уравнение

$$
\frac{\partial}{\partial t}\left(u_{x x x x}+u\right)-u_{x x}-u u_{x}=0
$$

известное как уравнение Розенау-Бюргерса. Несмотря на активное изучение данного уравнения, находящего свое применение в гидро- и электродинамике, к настоящему времени известно немного результатов о достаточных условиях разрушения его решений [1], [2]. В нашей работе получены достаточные условия существования непродолжаемого решения и его разрушения для серии начально-краевых задач для уравнения (1). При доказательстве разрушения использован метод пробных функций (нелинейной емкости) Похожаева и Митидиери [3]. Отметим, что к числу основных методов исследования разрушения решений относятся также энергетический метод Левина [4] и метод автомодельных режимов, основанный на различных методах сравнения и получивший свое развитие в работах Самарского, Галактионова, Курдюмова и Михайлова [5] (см. также работу [6]).

* Московский государственный университет им. М.В. Ломоносова, Москва, Россия. E-mail: a-panin@yandex.ru 


\section{2. ПОСТАНОВКА ЗАДАЧИ}

Рассмотрим серию начально-краевых задач следующего вида:

$$
\begin{gathered}
\frac{\partial}{\partial t}\left(u_{x x x x}+u\right)-u_{x x}-u u_{x}=0, \quad x \in[0, l], \quad t \in[0, T), \\
u(x, 0)=u_{0}(x), \quad x \in[0, l], \\
B_{j}[u(x, t)]=0, \quad t \in[0, T), \quad j=1,2,3,4,
\end{gathered}
$$

где $l=$ const - фиксированная длина промежутка, параметр $T \leqslant+\infty$ будет определен позднее, $u_{0}(x) \in C^{4}[0, l]$ - заданная функция, $B_{j}[u]$ - операторы граничных условий, каждый из которых является частным случаем выражения

$$
\sum_{k=0}^{3}\left(\left.\alpha_{k} \frac{\partial^{k} u}{\partial x^{k}}\right|_{x=0}+\left.\beta_{k} \frac{\partial^{k} u}{\partial x^{k}}\right|_{x=l}\right)
$$

Предполагается, что функция $u_{0}(x)$ удовлетворяет граничным условиям (4), таким образом, начальное и граничные условия задачи (2)-(4) согласованны. Мы будем рассматривать следующие варианты граничных условий:

$$
\begin{gathered}
u(0)=0, \quad u_{x}(0)=0, \quad u(l)=0, \quad l u_{x x x}(0)+u_{x x}(0)-u_{x x}(l)=0 ; \\
u(0)=0, \quad l u_{x}(0)-u(l)=0, \quad l u_{x x x}(0)+u_{x x}(0)=0, \quad u_{x x}(l)=0 ; \\
u(0)=0, \quad u_{x}(0)=0, \quad u(l)=0, \quad l u_{x x x}(0)+3 u_{x x}(0)=0 ; \\
u(0)=0, \quad u_{x}(0)=0, \quad u_{x x}(0)=0, \quad l^{3} u_{x x x}(0)-6 u(l)=0 .
\end{gathered}
$$

Как будет ясно из дальнейшего, результаты, аналогичные полученным в настоящей статье, можно получить для некоторого класса граничных условий, частным случаем которых являются (6)-(9).

Удобно применять следующие обозначения: для любой функции $z(x) \in C^{n}[0, l]$, $n=0,1,2, \ldots$, положим

$$
\|z\|_{n} \equiv \sum_{k=0}^{n} \sup _{x \in[0, l]}\left|\frac{d^{k} z(x)}{d x^{k}}\right| ;
$$

для любой функции $z(x)$, удовлетворяющей граничным условиям (4), определим линейный оператор

$$
\mathbb{L} z \equiv z_{x x x x}+z .
$$

Здесь и ниже для единообразия мы обозначаем производную по $x$ индексом, даже если речь идет об обыкновенной производной.

ОПРеДЕЛЕниЕ 1. Решением задачи (2)-(4) назовем функцию $u(t) \equiv u(x)(t)$ из класса $C^{1}\left([0, T) ; C^{4}[0, l]\right)$, удовлетворяющую (при каждом $\left.t \in[0, T)\right)$ в смысле сильного дифференцирования в пространстве $C^{4}[0, l]$ с нормой (10) уравнению

$$
\frac{d}{d t} \mathbb{L} u-u_{x x}-u u_{x}=0,
$$

а также начальному условию $u(0)=u_{0}(x)$ и (при каждом значении $\left.t \in[0, T)\right)$ граничным условиям (4). Величина $T$ может быть как конечной $(0<T<+\infty)$, так и бесконечной $(T=+\infty)$. 
Мы докажем, что для задач (2)-(4) верна следующая альтернатива: либо $T=+\infty$ (задача разрешима глобально по времени), либо

$$
T=T_{0}<+\infty, \quad \lim _{t \uparrow T_{0}}\|u(x)(t)\|_{C^{1}[0, l]}=+\infty
$$

т. е. решение разрушается за конечное время и реализуется режим blow-up. Также мы приведем достаточные условия режима blow-up.

\section{3. ЛОКАЛЬНАЯ РАЗРЕШИМОСТЬ}

3.1. Теорема о непродолжаемом решении. Рассмотрим абстрактную задачу Коши

$$
y^{\prime}(t)=A(y), \quad y(0)=y_{0}
$$

в банаховом пространстве $B$ с нормой $\|\cdot\|$ при $y_{0} \in B$. Под решением задачи Коши понимается функция $y \in C^{1}\left(\left[0, T_{0}\right) ; B\right)$, где $T_{0}<+\infty$ или $T_{0}=+\infty$, удовлетворяющая уравнению задачи (11), которое понимается в смысле сильного дифференцирования в банаховом пространстве $B$ при всех $t \in\left[0, T_{0}\right)$, и начальному условию $y(0)=y_{0}$.

Мы будем последовательно использовать следующую теорему.

ТЕорема 1. Пусть (нелинейный) оператор $A(y)$ локально липшиц-непрерывен, m.е. существует такая функиия $\mu:[0,+\infty) \rightarrow[0,+\infty)$, ограниченная на каждом ограниченном подмножестве луча $[0,+\infty)$, что для любых $z_{1}, z_{2} \in B$ выполнено неравенство

$$
\left\|A\left(z_{1}\right)-A\left(z_{2}\right)\right\| \leqslant \mu\left(\max \left\{\left\|z_{1}\right\|,\left\|z_{2}\right\|\right\}\right)\left\|z_{1}-z_{2}\right\| .
$$

Тогда существует и единственно решение $y(t), t \in\left[0, T_{0}\right)$, задачи $(1)$, для которого либо $T_{0}=+\infty$, либо

$$
T_{0}<+\infty, \quad \lim _{t \uparrow T_{0}}\|y(t)\|=+\infty ;
$$

любое другое решение может быть продолжено до указанного решения.

Решение, для которого $T_{0}=\infty$ или выполнено условие (13), мы будем называть непродолжаемым. Теорема 1 утверждает, в частности, что любое другое решение есть не что иное, как ограничение непродолжаемого решения на меньший временной промежуток.

Чтобы не разрывать изложение основного материала работы, мы вынесли доказательство теоремы 1 в конец статьи.

3.2. Обращение оператора $\mathbb{L}$. Допустимые граничные условия. Не конкретизируя пока выбор граничных условий, введем подпространство $Z_{0}^{4}$ функций $z(x) \in C^{4}[0, l]$, удовлетворяющих граничным условиям (4), и введем норму в этом подпространстве равенством $\|z\|_{Z_{0}^{4}}=\|z\|_{4}$ (см. (10)). Очевидно, подпространство $Z_{0}^{4}$ замкнуто по норме $\|\cdot\|_{4}$ и, таким образом, является банаховым пространством. Ранее был введен дифференциальный оператор

$$
\mathbb{L}: Z_{0}^{4} \rightarrow C[0, l], \quad \mathbb{L} z \equiv z_{x x x x}+z .
$$


Нам потребуется его обратный. Как известно, разрешимость краевой задачи

$$
\mathbb{L} z=f(x), \quad B_{j}[u(x, t)]=0, \quad j=1,2,3,4,
$$

зависит от граничных условий и длины $l$ отрезка. Поэтому обратимость оператора $\mathbb{L}$ нуждается в проверке. С этой целью будем искать решение задачи (14) в виде суммы решения $\bar{z}(x)$ задачи Коши для дифференциального уравнения $z_{x x x x}+z=f(x)$ с начальными условиями $z(0)=z_{x}(0)=z_{x x}(0)=z_{x x x}(0)=0$ и линейной комбинации линейно независимых частных решений $y_{1}(x), y_{2}(x), y_{3}(x), y_{4}(x)$ уравнения $z_{x x x}+$ $z=0$ :

$$
z(x)=\bar{z}(x)+\sum_{i=1}^{4} C_{i} y_{i}(x) \equiv \int_{0}^{x} K(x-s) f(s) d s+\sum_{i=1}^{4} C_{i} y_{i}(x)
$$

где $K(x-s)$ - функция Коши уравнения задачи (14), а частные решения имеют вид

$$
\begin{array}{ll}
y_{1}(x)=e^{x / \sqrt{2}} \cos \frac{x}{\sqrt{2}}, & y_{2}(x)=e^{-x / \sqrt{2}} \cos \frac{x}{\sqrt{2}} \\
y_{3}(x)=e^{x / \sqrt{2}} \sin \frac{x}{\sqrt{2}}, & y_{4}(x)=e^{-x / \sqrt{2}} \sin \frac{x}{\sqrt{2}} .
\end{array}
$$

Запишем условие того, что функция $z(x)$ удовлетворяет граничным условиям (4):

$$
B_{j}[\bar{z}(x)]+\sum_{i=1}^{4} C_{i} B_{j}\left[y_{i}(x)\right]=0, \quad j=1,2,3,4,
$$

где учтена линейность операторов $B_{j}$. Таким образом, относительно коэффициентов $C_{1}, C_{2}, C_{3}, C_{4}$ мы получаем систему линейных алгебраических уравнений с квадратной матрицей с элементами $B_{j}\left[y_{i}(x)\right], i, j=1,2,3,4$. Следовательно, оператор $\mathbb{L}$ обратим тогда и только тогда, когда

$$
\text { матрица }\left\{B_{j}\left[y_{i}(x)\right]\right\}_{i, j=\overline{1,4}} \text { невырожденна. }
$$

Именно это условие и определяет класс краевых задач, к которому применимы все рассуждения настоящего раздела. В частности, к этому классу относятся граничные условия (6)-(9). Чисто техническую проверку этого факта мы опускаем.

При выполнении условия (15) решение задачи (14) существует при любой правой части $f(x) \in C[0, l]$ и представимо в виде

$$
z(x)=\int_{0}^{l} G(x, s) f(s) d s
$$

3.3. Сведе́ние исходной задачи к задаче Коши в пространстве $Z_{0}^{4}$. Для удобства ссылок будем говорить о задаче (2)-(4) с теми или иными фиксированными (допустимыми) граничными условиями как о ЗАДАчЕ I. Для наших целей потребуется рассмотреть ряд других связанных с ней задач. 
Предположим, что $u(x)(t)$ - решение задачи I в смысле определения 1 . Отметим сразу, что тогда $u(x)(t) \in C^{1}\left([0, T) ; Z_{0}^{4}\right)$. Далее, если $u$ - решение задачи I в смысле определения 1 , то

$$
\frac{d}{d t} \mathbb{L} u=\mathbb{L} \frac{d u}{d t}
$$

знак обыкновенной производной здесь и всюду далее в работе подразумевает дифференцирование абстрактной функции со значениями в банаховом пространстве, а именно (в формуле (17)) в пространствах $C[0, l]$ и $Z_{0}^{4}$. Из определения 1 вытекает, что для решения $u$ задачи I выполняется равенство

$$
\mathbb{L} \frac{d u}{d t}=u_{x x}+u u_{x}
$$

где $u_{x x}+u u_{x}$ определяется естественным образом. В силу обратимости оператора $\mathbb{L}$ уравнение (18) равносильно следующему:

$$
\frac{d u}{d t}=\mathbb{G}\left(u_{x x}+u u_{x}\right)
$$

где $\mathbb{G} \equiv \mathbb{L}^{-1}: C[0, l] \rightarrow Z_{0}^{4}$ - оператор Грина задачи (14). Это ограниченный линейный оператор с конечной нормой $\|\mathbb{G}\|$ (это следует из существования функции Грина задачи (14), но конкретный вид этой функции нам не понадобится). Из приведенных выше рассуждений вытекает, что всякое решение в смысле определения 1 задачи I является решением задачи Коши

$$
\frac{d u}{d t}=\mathbb{G}\left(u_{x x}+u u_{x}\right), \quad u(0)=u_{0}(x), \quad u(x)(t) \in Z_{0}^{4} \text { при всех } t \in[0, T)
$$

в пространстве $Z_{0}^{4}$. Очевидно, верно и обратное: всякое решение задачи Коши (19), где $u_{0}(x) \in Z_{0}^{4}$, является решением задачи I в смысле определения 1 , поскольку для $u(x)(t) \in C^{1}\left([0, T) ; C^{4}[0, l]\right)$ верно тождество (17).

Итак, мы установили эквивалентность задачи I и задачи (19), которую в дальнейшем будем называть ЗАДАЧЕЙ II.

Введем (нелинейный) оператор

$$
A_{4}: Z_{0}^{4} \rightarrow Z_{0}^{4}, \quad A_{4}(u)=\mathbb{G}\left(u_{x x}+u u_{x}\right) .
$$

Легко видеть, что $u_{x x}+u u_{x} \in C^{2}[0, l] \subset C[0, l]$ при $u(x) \in C^{4}[0, l]$, тогда в силу свойств оператора Грина $\mathbb{G}$ - разрешающего оператора задачи $(14)$ - в самом деле $A_{4}(u) \in Z_{0}^{4}$. Теперь задача II может быть записана в этих обозначениях:

$$
\frac{d u}{d t}=A_{4}(u), \quad u(0)=u_{0}(x) .
$$

3.4. Локальная разрешимость задачи II. Докажем, что оператор $A_{4}(u)$ удовлетворяет условиям теоремы 1 для $B=Z_{0}^{4}$. Имеем

$$
\begin{aligned}
\left\|A_{4}(\bar{u})-A_{4}(\overline{\bar{u}})\right\|_{4} & =\left\|\mathbb{G}\left(\bar{u}_{x x}-\overline{\bar{u}}_{x x}\right)+\mathbb{G}\left(\bar{u} \bar{u}_{x}-\overline{\bar{u}} \overline{\bar{u}}_{x}\right)\right\|_{4} \leqslant \\
& \leqslant\|\mathbb{G}\|\left\|\bar{u}_{x x}-\overline{\bar{u}}_{x x}\right\|_{0}+\|\mathbb{G}\|\left\|\bar{u} \bar{u}_{x}-\overline{\bar{u}} \overline{\bar{u}}_{x}\right\|_{0} \leqslant \\
& \leqslant\|\mathbb{G}\|\|\bar{u}-\overline{\bar{u}}\|_{2}+\|\mathbb{G}\|\left\|\left(\bar{u} \bar{u}_{x}-\bar{u}_{\bar{u}}\right)+\left(\bar{u} \overline{\bar{u}}_{x}-\overline{\bar{u}} \overline{\bar{u}}_{x}\right)\right\|_{0} \leqslant
\end{aligned}
$$

4 Теоретическая и математическая физика, т. 177, № 1, 2013 г. 


$$
\begin{aligned}
& \leqslant\|\mathbb{G}\|\left(\|\bar{u}-\overline{\bar{u}}\|_{2}+\|\bar{u}\|_{0}\left\|\bar{u}_{x}-\overline{\bar{u}}_{x}\right\|_{0}+\left\|\overline{\bar{u}}_{x}\right\|_{0}\|\bar{u}-\overline{\bar{u}}\|_{0}\right) \leqslant \\
& \leqslant\|\mathbb{G}\|\left(\|\bar{u}-\overline{\bar{u}}\|_{4}+\|\bar{u}\|_{4}\|\bar{u}-\overline{\bar{u}}\|_{4}+\|\overline{\bar{u}}\|_{4}\|\bar{u}-\overline{\bar{u}}\|_{4}\right) \leqslant \\
& \leqslant\|\mathbb{G}\|\left(1+2 \max \left\{\|\bar{u}\|_{4},\|\overline{\bar{u}}\|_{4}\right\}\right)\|\bar{u}-\overline{\bar{u}}\|_{4},
\end{aligned}
$$

что и доказывает неравенство (12), в котором следует положить $\mu(\gamma)=\|\mathbb{G}\|(1+2 \gamma)$. Поэтому из теоремы 1 с учетом равносильности задач I и II непосредственно следует

ТЕОрема 2. Пусть граничные условия (4) удовлетворяют условию разрешимости (15). Тогда задача (19), а с ней и задача (2)-(4) локально (по времени) разрешимы при любой функиии $u_{0}(x) \in C^{4}[0, l]$, удовлетворяющей условиям (4).

3.5. Задача II в пространствах $Z^{2}$ и $Z^{1}$. Для наших целей необходимо рассмотреть более слабые постановки задачи II. Именно, нам требуется применить теорему 1 к пространству $B=Z^{1} \equiv C^{1}[0, l]$. Для этого мы установим следующие важные факты:

$1)$ оператор $\mathbb{G}$ можно расширить таким образом, что задачу II (т. е. задачу (19)) можно будет рассматривать в пространстве $Z^{1} \equiv C^{1}[0, l]$;

2 ) всякое решение задачи (19) в пространстве $Z^{1}$ при $u_{0}(x) \in Z_{0}^{4}$ является решением из пространства $Z_{0}^{4}$.

Эту программу мы реализуем в два этапа, последовательно понизив гладкость решения: сначала потребуем его принадлежность пространству $Z^{2} \equiv C^{2}[0, l]$, а затемтолько пространству $C^{1}[0, l]$. Перейдем к точным формулировкам.

Рассмотрим задачу

$$
\frac{d u}{d t}=\mathbb{G}\left(u_{x x}+u u_{x}\right), \quad u(0)=u_{0}(x), \quad u(x)(t) \in Z^{2} \text { при всех } t \in[0, T),
$$

которую мы назовем ЗАДАЧЕй ІІа. Легко видеть, что $u_{x x}+u u_{x} \in C[0, l]$ при $u \in Z^{2}$ и (в силу свойств оператора $\mathbb{G}) \mathbb{G}\left(u_{x x}+u u_{x}\right) \in Z_{0}^{4}$, т. е. в задаче $(21)$ оператор $\mathbb{G}$ может пониматься в том же смысле, что и выше. Однако нам необходимо уточнить его свойства.

Лемма 1. Для оператора

$$
A_{2 \rightarrow 4}: Z^{2} \rightarrow Z_{0}^{4}, \quad A_{2 \rightarrow 4}(u)=\mathbb{G}\left(u_{x x}+u u_{x}\right),
$$

верна ощенка

$$
\left\|A_{2 \rightarrow 4}(\bar{u})-A_{2 \rightarrow 4}(\overline{\bar{u}})\right\|_{4} \leqslant \nu\left(\max \left\{\|\bar{u}\|_{2},\|\overline{\bar{u}}\|_{2}\right\}\right)\|\bar{u}-\overline{\bar{u}}\|_{2}
$$

где $\nu(\gamma):[0,+\infty) \rightarrow[0,+\infty)$ - действительная функиия, ограниченная на каждом ограниченном подмножестве области определения.

ДоказАтЕЛьСтво. Подобно (20) имеем

$$
\begin{aligned}
\left\|A_{2 \rightarrow 4}(\bar{u})-A_{2 \rightarrow 4}(\overline{\bar{u}})\right\|_{4} & =\left\|\mathbb{G}\left(\bar{u}_{x x}-\overline{\bar{u}}_{x x}\right)+\mathbb{G}\left(\bar{u} \bar{u}_{x}-\overline{\bar{u}} \overline{\bar{u}}_{x}\right)\right\|_{4} \leqslant \\
& \leqslant\|\mathbb{G}\|\left\|\bar{u}_{x x}-\overline{\bar{u}}_{x x}\right\|_{0}+\|\mathbb{G}\|\left\|\bar{u} \bar{u}_{x}-\overline{\bar{u}} \overline{\bar{u}}_{x}\right\|_{0} \leqslant \\
& \leqslant\|\mathbb{G}\|\|\bar{u}-\overline{\bar{u}}\|_{2}+\|\mathbb{G}\|\left\|\left(\bar{u} \bar{u}_{x}-\bar{u} \overline{\bar{u}}_{x}\right)+\left(\bar{u} \overline{\bar{u}}_{x}-\overline{\bar{u}} \overline{\bar{u}}_{x}\right)\right\|_{0} \leqslant \\
& \leqslant\|\mathbb{G}\|\left(\|\bar{u}-\overline{\bar{u}}\|_{2}+\|\bar{u}\|_{0}\left\|\bar{u}_{x}-\overline{\bar{u}}_{x}\right\|_{0}+\left\|\overline{\bar{u}}_{x}\right\|_{0}\|\bar{u}-\overline{\bar{u}}\|_{0}\right) \leqslant
\end{aligned}
$$




$$
\begin{aligned}
& \leqslant\|\mathbb{G}\|\left(\|\bar{u}-\overline{\bar{u}}\|_{2}+\|\bar{u}\|_{2}\|\bar{u}-\overline{\bar{u}}\|_{2}+\|\overline{\bar{u}}\|_{2}\|\bar{u}-\overline{\bar{u}}\|_{2}\right) \leqslant \\
& \leqslant\|\mathbb{G}\|\left(1+2 \max \left\{\|\bar{u}\|_{2},\|\overline{\bar{u}}\|_{2}\right\}\right)\|\bar{u}-\overline{\bar{u}}\|_{2},
\end{aligned}
$$

что и доказывает неравенство $(22)$, в котором $\nu(\gamma)=\|\mathbb{G}\|(1+2 \gamma)$. Лемма доказана.

Теперь мы готовы доказать следующую теорему.

Теорема 3. Задачи II и IІа эквивалентны.

ДокАЗАтЕЛьство. В силу вложения $Z_{0}^{4} \subset Z^{2}$ и неравенства $\|\cdot\|_{2} \leqslant\|\cdot\|_{4}$ очевидно, что всякое решение задачи II является решением задачи ІІа. Докажем обратное утверждение. Заметим, что каждая из задач II, IІа эквивалентна интегральному уравнению

$$
u(t)=u_{0}+\int_{0}^{t} \mathbb{G}\left(u_{x x}(\tau)+u(\tau) u_{x}(\tau)\right) d \tau
$$

где, однако, в случае задачи II интеграл в (23) понимается в смысле интегрирования по Риману в банаховом пространстве $Z_{0}^{4}$, а в случае задачи IІа - в пространстве $Z^{2}$. Но если функция $u(t)$ дифференцируема в $Z^{2}$ при каждом $t \in[0, T)$, то она является функцией со значениями в $Z^{2}$, непрерывной по норме этого пространства. Тогда в силу леммы 1 и свойств оператора $\mathbb{G}$ подынтегральное выражение в (23) представляет собой функцию со значениями в $Z_{0}^{4}$, непрерывную по норме $Z_{0}^{4}$, и поэтому $Z_{0}^{4}$-интегрируемую. Следовательно, если интеграл в (23) существует в смысле $Z^{2}$, то он существует и в смысле $Z_{0}^{4}$. Поэтому всякое решение задачи IІа является решением задачи II.

Перейдем к самой слабой формулировке задачи: в пространстве $Z^{1} \equiv C^{1}[0, l]$. Чтобы рассмотреть задачу Коши типа задач II, IIa в пространстве $Z^{1}$, нам следует расширить оператор $\mathbb{G}$, первоначально определенный на $C[0, l]$, так, чтобы удалось определить оператор

$$
A_{1 \rightarrow 2}: Z^{1} \rightarrow Z^{2}, \quad A_{1 \rightarrow 2}(u)=\mathbb{G}\left(u_{x x}+u u_{x}\right) .
$$

Из способа построения функции Грина [7] следует, что для рассматриваемого оператора $\mathbb{L}$ с допустимыми граничными условиями (4) функция Грина, входящая в выражение (16), может быть представлена в виде

$$
G(x, s)= \begin{cases}G_{1}(x, s), & 0 \leqslant s<x, \\ G_{2}(x, s), & x<s \leqslant l,\end{cases}
$$

где $G_{1}(x, s), G_{2}(x, s)$ - функции, определенные при всех $x, s \in[0, l]$ и бесконечно дифференцируемые, причем при любом $x \in[0, l]$ верны равенства

$$
\begin{array}{cc}
G_{1}(x, x)=G_{2}(x, x), & \frac{\partial G_{1}}{\partial x}(x, x)=\frac{\partial G_{2}}{\partial x}(x, x), \\
\frac{\partial^{2} G_{1}}{\partial x^{2}}(x, x)=\frac{\partial^{2} G_{2}}{\partial x^{2}}(x, x), & \frac{\partial^{3} G_{1}}{\partial x^{3}}(x, x)-\frac{\partial^{3} G_{2}}{\partial x^{3}}(x, x)=1 .
\end{array}
$$


Дифференцируя эти равенства по $x$ в смысле полной производной, получаем в точках $(x, x), x \in[0, l]$, следующие соотношения:

$$
\begin{gathered}
\frac{\partial G_{1}}{\partial x}+\frac{\partial G_{1}}{\partial s}=\frac{\partial G_{2}}{\partial x}+\frac{\partial G_{2}}{\partial s}, \\
\frac{\partial^{2} G_{1}}{\partial x^{2}}+\frac{\partial^{2} G_{1}}{\partial s \partial x}=\frac{\partial^{2} G_{2}}{\partial x^{2}}+\frac{\partial^{2} G_{2}}{\partial s \partial x}, \\
\frac{\partial^{3} G_{1}}{\partial x^{3}}+\frac{\partial^{3} G_{1}}{\partial s \partial x^{2}}=\frac{\partial^{3} G_{2}}{\partial x^{3}}+\frac{\partial^{3} G_{2}}{\partial s \partial x^{2}}, \\
\frac{\partial^{4} G_{1}}{\partial x^{4}}+\frac{\partial^{4} G_{1}}{\partial s \partial x^{3}}=\frac{\partial^{4} G_{2}}{\partial x^{4}}+\frac{\partial^{4} G_{2}}{\partial s \partial x^{3}} .
\end{gathered}
$$

Сопоставляя (24) и (25), получаем в точках $(x, x), x \in[0, l]$, равенства

$$
\frac{\partial G_{1}}{\partial s}=\frac{\partial G_{2}}{\partial s}, \quad \frac{\partial^{2} G_{1}}{\partial s \partial x}=\frac{\partial^{2} G_{2}}{\partial s \partial x} .
$$

Рассмотрим $u \in C^{2}[0, l]$ и проведем следующее интегрирование по частям:

$$
\begin{aligned}
\mathbb{G} & \left(u_{x x}+u u_{x}\right)=\int_{0}^{l} G(x, s)\left(u_{x x}+u u_{x}\right) d s= \\
= & \int_{0}^{x} G_{1}(x, s)\left(u_{s s}(s)+\left(\frac{u^{2}}{2}\right)(s)\right) d s+\int_{x}^{l} G_{2}(x, s)\left(u_{s s}(s)+\left(\frac{u^{2}}{2}\right)(s)\right) d s= \\
= & \left.G_{1}(x, s)\left(u_{s}(s)+\frac{u^{2}}{2}(s)\right)\right|_{s=0} ^{s=x}-\int_{0}^{x} \frac{\partial G_{1}}{\partial s}(x, s)\left(u_{s}(s)+\frac{u^{2}}{2}(s)\right) d s+ \\
& +\left.G_{2}(x, s)\left(u_{s}(s)+\frac{u^{2}}{2}(s)\right)\right|_{s=x} ^{s=l}-\int_{x}^{l} \frac{\partial G_{2}}{\partial s}(x, s)\left(u_{s}(s)+\frac{u^{2}}{2}(s)\right) d s= \\
= & G_{1}(x, x)\left(u_{x}(x)+\frac{u^{2}}{2}(x)\right)-G_{1}(x, 0)\left(u_{x}(0)+\frac{u^{2}}{2}(0)\right)+ \\
& +G_{2}(x, l)\left(u_{x}(l)+\frac{u^{2}}{2}(l)\right)-G_{2}(x, x)\left(u_{x}(x)+\frac{u^{2}}{2}(x)\right)- \\
& -\int_{0}^{x} \frac{\partial G_{1}}{\partial s}(x, s)\left(u_{s}(s)+\frac{u^{2}}{2}(s)\right) d s-\int_{x}^{l} \frac{\partial G_{2}}{\partial s}(x, s)\left(u_{s}(s)+\frac{u^{2}}{2}(s)\right) d s .
\end{aligned}
$$

Правая часть полученного равенства имеет смысл и при $u \in C^{1}[0, l]$, причем в силу свойств (24) функции Грина для $u \in C^{1}[0, l]$ подстановки при $s=x$ взаимно уничтожаются, и мы можем записать

$$
\begin{aligned}
\mathbb{G}\left(u_{x x}\right. & \left.+u u_{x}\right)=G_{2}(x, l)\left(u_{x}(l)+\frac{u^{2}}{2}(l)\right)-G_{1}(x, 0)\left(u_{x}(0)+\frac{u^{2}}{2}(0)\right)- \\
& -\int_{0}^{x} \frac{\partial G_{1}}{\partial s}(x, s)\left(u_{s}(s)+\frac{u^{2}}{2}(s)\right) d s-\int_{x}^{l} \frac{\partial G_{2}}{\partial s}(x, s)\left(u_{s}(s)+\frac{u^{2}}{2}(s)\right) d s .
\end{aligned}
$$

Выражение в правой части имеет смысл при всех $u \in Z^{1}$, причем при всех $u \in Z^{2}$ его значение совпадает с ранее определенным. Итак, мы продолжили оператор

$$
u \mapsto \mathbb{G}\left(u_{x x}+u u_{x}\right),
$$


определенный первоначально для $u \in Z^{2}$, на $Z^{1}$. Убедимся, что полученный оператор (назовем его $A_{1 \rightarrow 2}$ ) переводит функции из $Z^{1}$ в функции из $Z^{2}$ и является локально липшиц-непрерывным как отображение из $Z^{1}$ в $Z^{2}$ и, следовательно, как отображение из $Z^{1}$ в $Z^{1}$. Для этого продифференцируем правую часть равенства (27) два раза по $x$, пользуясь свойствами (26) функции Грина:

$$
\begin{aligned}
\left(A_{1 \rightarrow 2}(u)\right)_{x}= & \frac{\partial G_{2}}{\partial x}(x, l)\left(u_{x}(l)+\frac{u^{2}}{2}(l)\right)-\frac{\partial G_{1}}{\partial x}(x, 0)\left(u_{x}(0)+\frac{u^{2}}{2}(0)\right)- \\
& -\frac{\partial G_{1}}{\partial s}(x, x)\left(u_{x}(x)+\frac{u^{2}}{2}(x)\right)-\int_{0}^{x} \frac{\partial^{2} G_{1}}{\partial x \partial s}(x, s)\left(u_{s}(s)+\frac{u^{2}}{2}(s)\right) d s+ \\
& +\frac{\partial G_{2}}{\partial s}(x, x)\left(u_{x}(x)+\frac{u^{2}}{2}(x)\right)-\int_{x}^{l} \frac{\partial^{2} G_{2}}{\partial x \partial s}(x, s)\left(u_{s}(s)+\frac{u^{2}}{2}(s)\right) d s= \\
= & \frac{\partial G_{2}}{\partial x}(x, l)\left(u_{x}(l)+\frac{u^{2}}{2}(l)\right)-\frac{\partial G_{1}}{\partial x}(x, 0)\left(u_{x}(0)+\frac{u^{2}}{2}(0)\right)- \\
& -\int_{0}^{x} \frac{\partial^{2} G_{1}}{\partial x \partial s}(x, s)\left(u_{s}(s)+\frac{u^{2}}{2}(s)\right) d s-\int_{x}^{l} \frac{\partial^{2} G_{2}}{\partial x \partial s}(x, s)\left(u_{s}(s)+\frac{u^{2}}{2}(s)\right) d s
\end{aligned}
$$

и далее

$$
\begin{aligned}
\left(A_{1 \rightarrow 2}(u)\right)_{x x}=\frac{\partial^{2} G_{2}}{\partial x^{2}}(x, l)\left(u_{x}(l)+\frac{u^{2}}{2}(l)\right)-\frac{\partial^{2} G_{1}}{\partial x^{2}}(x, 0)\left(u_{x}(0)+\frac{u^{2}}{2}(0)\right)- \\
-\frac{\partial^{2} G_{1}}{\partial x \partial s}(x, x)\left(u_{x}(x)+\frac{u^{2}}{2}(x)\right)-\int_{0}^{x} \frac{\partial^{3} G_{1}}{\partial x^{2} \partial s}(x, s)\left(u_{s}(s)+\frac{u^{2}}{2}(s)\right) d s+ \\
\quad+\frac{\partial^{2} G_{2}}{\partial x \partial s}(x, x)\left(u_{x}(x)+\frac{u^{2}}{2}(x)\right)-\int_{x}^{l} \frac{\partial^{3} G_{2}}{\partial x^{2} \partial s}(x, s)\left(u_{s}(s)+\frac{u^{2}}{2}(s)\right) d s= \\
=\frac{\partial^{2} G_{2}}{\partial x^{2}}(x, l)\left(u_{x}(l)+\frac{u^{2}}{2}(l)\right)-\frac{\partial^{2} G_{1}}{\partial x^{2}}(x, 0)\left(u_{x}(0)+\frac{u^{2}}{2}(0)\right)- \\
\quad-\int_{0}^{x} \frac{\partial^{3} G_{1}}{\partial x^{2} \partial s}(x, s)\left(u_{s}(s)+\frac{u^{2}}{2}(s)\right) d s-\int_{x}^{l} \frac{\partial^{3} G_{2}}{\partial x^{2} \partial s}(x, s)\left(u_{s}(s)+\frac{u^{2}}{2}(s)\right) d s .
\end{aligned}
$$

Из вида полученных выражений в силу ограниченности входящих в него производных функций $G_{1}(x, s), G_{2}(x, s)$ на компактных множествах ясно, что оператор $A_{1 \rightarrow 2}(u)$ является локально липшиц-непрерывным как отображение из $Z^{1}$ в $Z^{2}$ и тем более как отображение из $Z^{1}$ в $Z^{1}$. Это позволяет рассмотреть ЗАДАчУ ІІб:

$$
\frac{d u}{d t}=\mathbb{G}\left(u_{x x}+u u_{x}\right), \quad u(0)=u_{0}(x), \quad u(x)(t) \in Z^{1} \text { при всех } t \in[0, T) .
$$

Аналогично случаю теоремы 3 можно доказать следующую теорему.

ТеОрема 4. Задачи ІІа и ІІб эквивалентны.

С другой стороны, в силу локальной липшиц-непрерывности оператора $A_{1 \rightarrow 2}(u)$ как отображения из $Z^{1}$ в $Z^{1}$ можно применить к задаче ІІб теорему 1.

Подытожим результаты, полученные в этом разделе. Мы установили, что задачи II, IІа, ІІб эквивалентны друг другу и удовлетворяют условиям теоремы 1, поэтому непродолжаемые решения каждой из этих задач существуют, единственны и совпадают друг с другом. Конечно, здесь было существенно условие $u_{0}(x) \in C^{4}[0, l]$. Необходимые для дальнейшего следствия из этого факта оформим в виде теоремы. 
ТеОРема 5. Имеют место следующие утверждения.

1. Задача ІІб однозначно разрешима на некотором максимальном промежутке $[0, T)$.

2. Если $T<+\infty$, то справедливо соотношение

$$
\lim _{t \rightarrow T-0}\|u(x)(t)\|_{1}=+\infty
$$

3. Задача II, а с ней и исходная задача I разрешимы на том же самом промежутке $[0, T)$.

3.6. Разрушение решений. Как доказано выше, для решения задачи I выполняется уравнение (18) или, эквивалентно, уравнение

$$
\left(u^{\prime}\right)_{x x x x}+u^{\prime}-u_{x x}-u u_{x}=0 .
$$

Рассмотрим сначала граничные условия (6) и (7), а затем - условия (8) и (9).

3.6.1. Случай граничных условий (6) и (7). Предварительно докажем лемму.

ЛЕмма 2. Пусть $T=$ const, $0<T<\infty$, и функиия $J(t)$ удовлетворяет следующим условиям:

1) $J(t) \in C[0, T) \cap C^{1}(0, T)$;

2) $J(0)>0$;

3) найдется $a>0$ такое, что для всех $t \in(0, T)$ выполнено неравенство

$$
\frac{d J}{d t} \geqslant a J^{2}
$$

Тогда при всех $t \in[0, T)$

$$
J(t) \geqslant \frac{J(0)}{1-a J(0) t}
$$

ДокАЗАТЕЛЬСтво. В силу условий 2,3 леммы имеем $J(t) \geqslant J(0)>0$ при всех значениях $t \in[0, T)$. Следовательно,

$$
\frac{J^{\prime}}{J^{2}} \geqslant a, \quad-\left.\frac{1}{J}\right|_{t=0} ^{t=t} \geqslant a t, \quad \frac{1}{J(0)}-\frac{1}{J(t)} \geqslant a t, \quad \frac{1}{J(t)} \leqslant \frac{1}{J(0)}-a t,
$$

таким образом,

$$
J(t) \geqslant \frac{J(0)}{1-a J(0) t} \quad \text { при } \quad t \in\left(0, \frac{1}{a J(0)}\right) .
$$

Из последнего неравенства и условия 1 леммы следует, что $T \leqslant 1 / a J(0)$, в результате неравенство (30) выполнено на всем промежутке существования функции $J(t)$.

СлЕДСТВИЕ 1. При выполнении условий леммы с необходимостъю получаем следующую оценку: $T \leqslant 1 /$ аJ $(0)$. 
Заметим, что при выполнении граничных условий (6) или (7) верны следующие тождества:

$$
\begin{aligned}
\int_{0}^{l}(l-x)\left(u^{\prime}\right)_{x x x x} d x & =\left.(l-x)\left(u^{\prime}\right)_{x x x}\right|_{0} ^{l}+\int_{0}^{l}\left(u^{\prime}\right)_{x x x} d x= \\
& =-l\left(u^{\prime}\right)_{x x x}(0)+\left(u^{\prime}\right)_{x x}(l)-\left(u^{\prime}\right)_{x x}(0)=0 \\
\int_{0}^{l}(l-x) u_{x x} d x & =\left.(l-x) u_{x}\right|_{0} ^{l}+\int_{0}^{l} u_{x} d x=-l u_{x}(0)+u(l)-u(0)=0, \\
\int_{0}^{l}(l-x) u u_{x} d x & =\frac{1}{2} \int_{0}^{l}(l-x)\left(u^{2}\right)_{x} d x=\left.\frac{1}{2}(l-x) u^{2}\right|_{0} ^{l}+\frac{1}{2} \int_{0}^{l} u^{2} d x= \\
& =-\frac{1}{2} l u^{2}(0)+\frac{1}{2} \int_{0}^{l} u^{2} d x=\frac{1}{2} \int_{0}^{l} u^{2} d x
\end{aligned}
$$

Домножим уравнение (29) при каждом $t \in[0, T)$ на функцию $l-x$ и проинтегрируем по отрезку $[0, l]$. Тогда с учетом тождеств $(31)$, а также тождества

$$
\int_{0}^{l}(l-x) u^{\prime} d x=\frac{d}{d t} \int_{0}^{l}(l-x) u d x
$$

вытекающего из свойств гладкости функции $u(x)(t)$, заданных в определении 1 , получим из (29) дифференциальное соотношение

$$
\frac{d}{d t} \int_{0}^{l}(l-x) u d x=\frac{1}{2} \int_{0}^{l} u^{2} d x .
$$

Применяя теперь неравенство Коши-Буняковского для интегралов, получим

$$
\left(\int_{0}^{l}(l-x) u d x\right)^{2} \leqslant \int_{0}^{l}(l-x)^{2} d x \cdot \int_{0}^{l} u^{2} d x=\frac{l^{3}}{3} \int_{0}^{l} u^{2} d x
$$

или, если положить $J(t)=\int_{0}^{l}(l-x) u d x$,

$$
\frac{1}{2} \int_{0}^{l} u^{2} d x \geqslant \frac{3}{2 l^{3}} J^{2}
$$

С учетом этого неравенства из (32) получаем

$$
\frac{d}{d t} J(t) \geqslant \frac{3}{2 l^{3}} J^{2}
$$

Таким образом, если потребовать

$$
\int_{0}^{l}(l-x) u_{0}(x) d x>0
$$

функция $J(t)$ удовлетворяет условиям леммы 2 , в которой следует взять $a=3 / 2 l^{3}$. Из следствия 1 вытекает, что при условии (33) решение задачи I может существовать лишь на ограниченном промежутке времени $t$. 
Итак, доказана

ТеОРема 6. При выполнении условия (33) решение задачи (2)-(4) может существовать лишь на ограниченном промежутке времени $[0, T)$, где

$$
T \leqslant \frac{2 l^{3}}{3 J(0)}, \quad J(0)=\int_{0}^{l}(l-x) u_{0}(x) d x .
$$

3.6.2. Случай граничных условий (8) и (9). Предварительно докажем лемму.

ЛЕмма 3. Пусть функиия $J(t)$ удовлетворяет следующим условиям:

1) $J(t) \in C[0, T) \cap C^{1}(0, T)$;

2) найдутся $k>0$ и $m>0$ такие, что при всех $t \in(0, T)$, где $0<T \leqslant+\infty$, имеет место неравенство

$$
\frac{d J}{d t} \geqslant k^{2} J^{2}-m^{2}
$$

3) справедливо неравенство $J(0)>m / k$.

Тогда при всех $t \in[0, T)$

$$
J(t) \geqslant \frac{m\left(1+A_{0} e^{2 k m t}\right)}{k\left(1-A_{0} e^{2 k m t}\right)}, \quad \text { əде } \quad A_{0}=\frac{k J(0)-m}{k J(0)+m} .
$$

ДокАзАтЕльство. Заметим прежде всего, что при всех $t \in[0, T)$ выполняется неравенство $J(t)>m / k$. В самом деле, если оно нарушается на некотором множестве значений $T^{*}$, положим $t^{*}=\inf T^{*}$. В силу условий 1,3 леммы в этом случае $t^{*}>0$ и $J\left(t^{*}\right)=m / k$. В силу задачи о среднем существует такое $t^{* *} \in\left(0, t^{*}\right)$, что $J^{\prime}\left(t^{* *}\right)<0$. Но по определению $t^{*}$ имеем $J(t)>m / k$ при всех $t \in\left[0, t^{*}\right)$, и это в силу условия 2 леммы гарантирует, что $J^{\prime}\left(t^{* *}\right)>0$. Полученное противоречие доказывает, что $J(t)>m / k$ при всех $t \in[0, T)$.

Теперь мы можем проинтегрировать неравенство (35), получим

$$
\left.\frac{1}{2 k m} \ln \frac{k J-m}{k J+m}\right|_{J(0)} ^{J(t)} \geqslant t,
$$

что после преобразований сводится к неравенству (36).

СлеДСТвИЕ 2. В условиях леммы с необходимостью выполняется следующая оченка:

$$
T \leqslant \frac{1}{2 k m} \ln \frac{1}{A_{0}}
$$

В случае граничных условий (8) или (9) аналогично (31) получим

$$
\begin{aligned}
\int_{0}^{l}(l-x)^{3}\left(u^{\prime}\right)_{x x x x} d x & =0, \\
\int_{0}^{l}(l-x)^{3} u_{x x} d x & =6 \int_{0}^{l}(l-x) u d x, \\
\int_{0}^{l}(l-x)^{3} u u_{x} d x & =\frac{3}{2} \int_{0}^{l}(l-x)^{2} u^{2} d x .
\end{aligned}
$$


Домножим уравнение (29) при каждом $t \in[0, T)$ на функцию $(l-x)^{3}$ и проинтегрируем по отрезку $[0, l]$. Тогда с учетом тождеств $(37)$, а также тождества

$$
\int_{0}^{l}(l-x)^{3} u^{\prime} d x=\frac{d}{d t} \int_{0}^{l}(l-x)^{3} u d x
$$

вытекающего из свойств гладкости функции $u(x)(t)$, заданных в определении 1 , получаем дифференциальное соотношение

$$
\frac{d}{d t} \int_{0}^{l}(l-x)^{3} u d x=6 \int_{0}^{l}(l-x) u d x+\frac{3}{2} \int_{0}^{l}(l-x)^{2} u^{2} d x .
$$

Добавив в левую часть под знак производной по времени не зависящее от времени выражение, определим функцию $J(t)$ выражением

$$
J(t)=\int_{0}^{l}(l-x)^{2}[(l-x) u+2] d x .
$$

Применяя неравенство Коши-Буняковского для интегралов, получим

$$
\begin{aligned}
J^{2}(t) & \leqslant \int_{0}^{l}(l-x)^{4} d x\left[\int_{0}^{l}(l-x)^{2} u^{2} d x+4 \int_{0}^{l}(l-x) u d x+4 \int_{0}^{l} d x\right]= \\
& =\frac{l^{5}}{5}\left[\int_{0}^{l}(l-x)^{2} u^{2} d x+4 \int_{0}^{l}(l-x) u d x+4 l\right] .
\end{aligned}
$$

Сравнивая правую часть последней формулы с правой частью равенства (38), получаем следствие из соотношения (38):

$$
\frac{d J}{d t} \geqslant \frac{15}{2 l^{5}} J^{2}-6 l,
$$

что совпадает с неравенством (35) при $k^{2}=15 / 2 l^{5}$ и $m^{2}=6 l$.

Таким образом, доказана

ТЕОРема 7. При условии

$$
J(0) \equiv \int_{0}^{l}(l-x)^{2}\left[(l-x) u_{0}(x)+2\right] d x>\frac{m}{k}, \quad \text { əде } \quad m=\sqrt{6 l}, \quad k=\sqrt{\frac{15}{2 l^{5}}},
$$

решение $u(x)(t)$ задачи I существует только на ограниченном промежутке $[0, T)$, где

$$
T \leqslant \frac{1}{2 k m} \ln \frac{1}{A_{0}}
$$

а константа $A_{0}$ (зависящая от $m, k$ и $\left.J(0)\right)$ определена в (36).

3.6.3. Разрушение решения по норме пространства $C^{1}[0, l]$. В данном пункте (независимо от решения проблемы существования решения) мы построим достаточные условия разрушения решения задачи I для различных граничных условий. Как было показано выше (см. теорему 5), задачи I, II и ІІб однозначно разрешимы на некотором (конечном или бесконечном) промежутке времени $t$ ненулевой длины и решение любой из них (независимо от способа его построения) является решением всех 
других на том же самом промежутке. Следовательно, если решение задачи I (или, что то же самое, задачи II) не существует глобально по времени, то не существует глобально по времени и решение "ослабленной" задачи ІІб. Но для задачи ІІб, как установлено выше, выполняются все условия теоремы 1. Следовательно, из глобальной неразрешимости этой задачи следует согласно соотношению (13), что

$$
\lim _{t \uparrow T}\|u(x)(t)\|_{C^{1}[0, l]}=+\infty
$$

как только выполнены условия глобальной неразрешимости задачи I (т.е. условие (33) в случае граничных условий (6), (7) и условие (40) в случае граничных условий (8), (9)).

Основные результаты работы сформулируем в итоговой теореме.

Теорема 8. Имеют место следующие утверждения.

1. Задача (2)-(4) однозначно разрешима (по крайней мере локально по времени) при любых граничных условиях (4) вида (5), удовлетворяющих требованию (15).

2. В случае выбора граничных условий (4) в виде (6) или (7) при выполнении условия (33), а в случае выбора граничных условий в виде (8) или (9) при выполнении условия (40) решение задачи (2)-(4) существует лишь на ограниченном промежутке времени (оценки времени разрушения $T$ даются бормулами (34) или (41) соответственно), причем имеет место предельное соотношение (42).

ЗАмЕчАниЕ 1. Из приведенных выше рассуждений видно, что, помимо условий (6)-(9), результаты сохраняют силу для любых граничных условий, допустимых в смысле требования (15) и позволяющих получить тождества (31) или (37).

\section{4. ДОКАЗАТЕЛЬСТВО ТЕОРЕМЫ 1}

Напомним, что мы ищем решение абстрактной задачи Коши

$$
y^{\prime}(t)=A(y), \quad y(0)=y_{0}
$$

в банаховом пространстве $B$ при $y_{0} \in B$ в классе $C^{1}\left(\left[0, T_{0}\right) ; B\right)$. При этом мы считаем, что для оператора $A$ выполнено условие локальной липшиц-непрерывности:

$$
\left\|A\left(z_{1}\right)-A\left(z_{2}\right)\right\| \leqslant \mu\left(\max \left(\left\|z_{1}\right\|,\left\|z_{2}\right\|\right)\right)\left\|z_{1}-z_{2}\right\|, \quad z_{1}, z_{2} \in B
$$

Мы докажем, что при этих условиях существует и единственно решение, для которого либо $T_{0}=+\infty$, либо

$$
T_{0}<+\infty, \quad \lim _{t \uparrow T_{0}}\|y(t)\|=+\infty
$$

(такое решение мы называем непродолжаемым).

1. Прежде всего докажем локальную разрешимость задачи Коши (43). Заметим, что следствием (43) является интегральное уравнение

$$
y(t)=y_{0}+\int_{0}^{t} A(y(\tau)) d \tau,
$$


или $y=\mathbb{A}(y)$, где $\mathbb{A}(z) \equiv y_{0}+\int_{0}^{t} A(z(\tau)) d \tau$ - оператор, действующий в банаховом пространстве $\mathbb{B}=C([0, T] ; B)$, а величина $T>0$ будет определена ниже. Интеграл в правой части равенства (46) имеет смысл для всех $y(t) \in \mathbb{B}$, поскольку подынтегральное выражение непрерывно как композиция непрерывных отображений. Положим также

$$
\|z\|_{\mathbb{B}}=\sup _{t \in[0, T]}\|z(t)\| .
$$

Мы докажем локальную разрешимость уравнения (46) в классе $C\left(\left[0, T_{0}\right) ; B\right)$ и после этого покажем, что каждое такое решение является решением задачи (43), принадлежащим пространству $C^{1}\left(\left[0, T_{0}\right) ; B\right)$.

Зафиксируем произвольное $R>0$ и рассмотрим замкнутое подмножество

$$
\mathbb{B}_{R}=\left\{y(t) \in \mathbb{B}:\left\|y-y_{0}\right\|_{\mathbb{B}} \leqslant R\right\} .
$$

В силу общих свойств метрических пространств множество $\mathbb{B}_{R}$ является полным метрическим пространством относительно расстояния, порожденного нормой пространства $\mathbb{B}$. Теперь укажем, как выбрать $T>0$ так, чтобы оператор $\mathbb{A}$ :

1) не выводил из множества $\mathbb{B}_{R}$,

2) являлся на этом множестве сжимающим.

Заметим, что в силу условия (44) верна оценка

$$
\|A(z)\| \leqslant \mu(\max \{\|z\|, 0\})\|z-0\|=\mu(\|z\|)\|z\|,
$$

а тогда для всех $y(t) \in \mathbb{B}_{R}$ в силу (47) и (48)

$$
\begin{aligned}
\left\|\int_{0}^{t} A(y(\tau)) d \tau\right\|_{\mathbb{B}} & \equiv \sup _{t \in[0, T]}\left\|\int_{0}^{t} A(y(\tau)) d \tau\right\| \leqslant \\
& \leqslant \int_{0}^{T}\|A(y(\tau))\| d \tau \leqslant T \cdot \sup _{r \in\left[0,\left\|y_{0}\right\|+R\right]} \mu(r) \cdot\left(\left\|y_{0}\right\|+R\right) .
\end{aligned}
$$

Поскольку функция $\mu(\cdot)$ ограничена на любом ограниченном подмножестве в $\mathbb{R}_{+}$, можно выбрать такое $T>0$, что правая часть последнего неравенства будет меньше $R$, и тем самым требование 1 к оператору $\mathbb{A}$ будет выполнено.

Теперь обеспечим выполнение требования 2. Имеем с учетом (44) и (47) для любых $y_{1}(t), y_{2}(t) \in \mathbb{B}_{R}$

$$
\begin{aligned}
& \left\|\int_{0}^{t} A\left(y_{1}(\tau)\right) d \tau-\int_{0}^{t} A\left(y_{2}(\tau)\right) d \tau\right\|_{\mathbb{B}} \leqslant \int_{0}^{T}\left\|A\left(y_{1}(\tau)\right)-A\left(y_{2}(\tau)\right)\right\| d \tau \leqslant \\
& \quad \leqslant T \cdot \sup _{r \in\left[0,\left\|y_{0}\right\|+R\right]} \mu(r) \cdot \sup _{t \in[0, T]}\left\|y_{1}(t)-y_{2}(t)\right\|=T \cdot \sup _{r \in\left[0,\left\|y_{0}\right\|+R\right]} \mu(r) \cdot\left\|y_{1}-y_{2}\right\|_{\mathbb{B}} .
\end{aligned}
$$

Теперь мы уменьшим, если понадобится, $T$ настолько, чтобы выполнялось условие

$$
T \cdot \sup _{r \in\left[0,\left\|y_{0}\right\|+R\right]} \mu(r) \leqslant \frac{1}{2} \text {. }
$$

Итак, при выборе $T$ из условий

$$
T \cdot \sup _{r \in\left[0,\left\|y_{0}\right\|+R\right]} \mu(r) \cdot\left(\left\|y_{0}\right\|+R\right) \leqslant R, \quad T \cdot \sup _{r \in\left[0,\left\|y_{0}\right\|+R\right]} \mu(r) \leqslant \frac{1}{2}
$$


оператор $\mathbb{A}$ не выводит из множества $\mathbb{B}_{R}$ и является на этом множестве сжимающим отображением.

По теореме о неподвижной точке при таком выборе $T$ уравнение (46) имеет единственное решение в пространстве $\mathbb{B}_{R}$. Но тогда подынтегральное выражение в (46) непрерывно как композиция непрерывных отображений и, таким образом, функция $y(t)$ представима в виде интеграла с переменным верхним пределом от непрерывной функции. Следовательно, $y(t) \in C^{1}([0, T] ; B)$ и является решением задачи Коши (43) на промежутке $[0, T)$. Локальная разрешимость задачи (43) доказана.

Приведенное рассуждение показывает также единственность решения задачи (43) независимо от способа его получения. В самом деле, рассмотрев интегральное уравнение (46) с началом в точке $T_{1}$, за которой нарушается единственность, и проведя рассуждения, аналогичные приведенным выше (с очевидной заменой $0 \mapsto T_{1}$ и подходящим выбором $R$ и $T$ ), мы сразу же приходим к противоречию с теоремой о неподвижной точке.

2. Теперь докажем, что отсутствие глобального по $t$ решения влечет стремление решения к бесконечности. Докажем сначала, что если решение не существует глобально по времени, то выполнено более слабое, чем (45), предельное соотношение

$$
\underset{t \uparrow T_{0}}{\limsup }\|y(t)\|=+\infty
$$

Рассмотрим всевозможные функции $z(t) \in C^{1}([0,+\infty) ; B)$. Если среди них существует функция, удовлетворяющая задаче (43) при всех $t \geqslant 0$, то утверждение теоремы установлено. Если же такой функции не существует, положим

$$
T_{0} \equiv \sup \left\{T: \exists \text { решение задачи }(43) \text { из класса } C^{1}([0, T] ; B)\right\} \text {. }
$$

В силу предыдущих рассуждений $T_{0}>0$. С учетом установленной выше единственности решения возможны два случая:

1) существует решение из класса $C^{1}\left(\left[0, T_{0}\right] ; B\right)$,

2) решение существует лишь в классе $C^{1}\left(\left[0, T_{0}\right) ; B\right)$.

В первом случае, очевидно, все условия теоремы выполняются для задачи Коши, аналогичной (43), с начальными данными в точке $t=T_{0}$ вместо $t=0$. Следовательно, в силу п. 1 доказательства решение может быть продолжено по $t$ хотя бы на небольшой отрезок. При этом, очевидно,

$$
\lim _{t \uparrow T_{0}} y^{\prime}(t)=\lim _{t \downarrow T_{0}} y^{\prime}(t)=A\left(T_{0}\right)\left(y\left(T_{0}\right)\right),
$$

поэтому полученное "составное" решение непрерывно дифференцируемо на всем промежутке существования. Это противоречит определению величины $T_{0}$, следовательно, первый случай исключается.

Во втором случае соотношение (49) может иметь или не иметь место. Если оно выполнено, то остается лишь вывести из него соотношение (45). В противном случае

$$
\underset{t \uparrow T_{0}}{\limsup }\|y\|=M<+\infty
$$


откуда с учетом оценки (48) и свойств функции $\mu$ имеем

$$
\underset{t \uparrow T_{0}}{\limsup }\|A(y)\|=\mathcal{L}<+\infty
$$

т. е. действительная функция $\|A(y(t))\|$, а с нею и $\left\|y^{\prime}(t)\right\|$ (в силу уравнения (43)) ограничены. Но тогда абстрактная функция $y(t)$ липшиц-непрерывна в левой полуокрестности точки $T_{0}$ с константой $\mathcal{L}$ и, следовательно, эту функцию можно продолжить по непрерывности в точку $T_{0}$ в силу выполнения критерия Коши существования предела функции. Рассуждая аналогично предыдущему, устанавливаем из интегрального уравнения (46), что производная $y^{\prime}(t)$ существует и непрерывна вплоть до значения $T_{0}$ включительно. Таким образом, мы продолжили решение до $y(t) \in C^{1}\left(\left[0, T_{0}\right] ; B\right)$ и пришли к случаю 1 , невозможность которого установлена ранее. Итак, в случае отсутствия глобального по времени решения возможен лишь случай 2 и при этом должно выполняться предельное соотношение (49).

Выведем теперь из этого факта соотношение (45). Предположим, что оно не имеет места, или, другими словами,

$$
\exists M_{1}>0: \forall \delta>0 \quad \exists t \in\left(T_{0}-\delta, T_{0}\right), \quad \text { при котором }\|y(t)\| \leqslant M_{1} .
$$

Заметим, что в силу оценки (48) и свойств функции $\mu$ существует $E>0$ такое, что при всех $z \in B,\|z\| \leqslant 2 M_{1}$, верно неравенство

$$
\|A(z)\| \leqslant E .
$$

Выберем теперь произвольное $\delta \in\left(0, M_{1} / E\right)$. Пусть $t_{1} \in\left(T_{0}-\delta, T_{0}\right)$ выбрано из условия (50), т. е. выполнено условие

$$
\left\|y\left(t_{1}\right)\right\| \leqslant M_{1}
$$

В силу соотношения (49) и непрерывности $\|y(t)\|$ как функции аргумента $t$ существует $t_{2} \in\left(t_{1}, T\right)$ такое, что

$$
\left\|y\left(t_{2}\right)\right\|=2 M_{1}, \quad\|y(t)\| \leqslant 2 M_{1} \text { при } t \in\left[t_{1}, t_{2}\right) .
$$

Из последнего неравенства, неравенства (51) и уравнения задачи Коши (43) следует, что $\left\|y^{\prime}(t)\right\| \leqslant E$ при всех $t \in\left[t_{1}, t_{2}\right]$. Однако отсюда и из соотношений

$$
\left\|y\left(t_{1}\right)\right\| \leqslant M_{1}, \quad\left\|y\left(t_{2}\right)\right\|=2 M_{1}, \quad t_{2}-t_{1}<\delta<\frac{M_{1}}{E} .
$$

получаем противоречие: с одной стороны, $\left\|y\left(t_{2}\right)\right\|-\left\|y\left(t_{1}\right)\right\|<\left(M_{1} / E\right) E=M_{1}$, а с другой - $\left\|y\left(t_{2}\right)\right\|-\left\|y\left(t_{1}\right)\right\| \geqslant 2 M_{1}-M_{1}=M_{1}$. Полученное противоречие завершает доказательство теоремы.

Благодарности. Автор выражает свою глубокую признательность М. О. Корпусову за постановку задачи и А. Шеню за консультацию, касающуюся теоремы 1. 


\section{Список литературы}

[1] М. О. Корпусов, ТМФ, 170:3 (2012), 342-349.

[2] M. O. Korpusov, Nonlin. Anal., 75:4 (2012), 1737-1743.

[3] Э. Л. Митидиери, С. И. Похожаев, Тр. МИАН, 234 (2001), 3-383.

[4] H. A. Levine, Arch. Rational Mech. Anal., 51:3 (1973), 371-386.

[5] А.А. Самарский, В.А. Галактионов, С.П. Курдюмов, А.П. Михайлов, Режимы $c$ обострением в задачах для квазилинейных параболических уравнений, Наука, М., 1987.

[6] В. А. Галактионов, С.И. Похожаев, Ж. вычисл. матем. и матем. физ., 48:10 (2008), 1819-1846.

[7] М. А. Наймарк, Линейнъе дифференииалъные операторы, Наука, М., 1969.

Поступила в редакцию 29.01.2013 